\title{
Composición, Análisis Termofísico y Sensorial de Frutos Colombianos. Parte 2: Acerola (Malpighia emarginata L.)
}

\author{
Guillermo Arrázola ${ }^{(1)}$, Armando Alvis(1) y Manuel Páez ${ }^{(2)}$ \\ (1) Universidad de Córdoba. Programa de Ingeniería de Alimentos. Facultad de Ingenierías. Grupo de \\ Investigación Procesos y Agroindustria de vegetales. Carrera 6 No76-103, Km 3, vía Cereté. Córdoba- \\ Colombia. (e-mail: guillermo.arrazola@ua.es). \\ (2) Universidad de Córdoba. Programa de Química. Facultad de Ciencias básicas. Km 3 Vía Cereté. \\ Córdoba, Colombia.
}

Recibido Oct. 14, 2013; Aceptado Dic. 13, 2013; Versión final recibida Ene. 29, 2014

\begin{abstract}
Resumen
El objetivo del trabajo fue estudiar los contenidos de humedad, carbohidratos y cenizas en acerolas (Malpighia emarginata L) en el departamento de Córdoba, así como analizar las propiedades termofísicas y sensoriales. Las propiedades termofísicas, como conductividad y difusividad térmica, calor específico y densidad, fueron determinadas en el fruto de acuerdo a la composición bromatológica de la acerola y comparándolas con el software DEPROTER. Los valores cenizas y humedad en frutos, no mostraron diferencias significativas en las regiones del alto y medio Sinú, los carbohidratos, presentaron diferencias significativas $(p<0.05)$ para las tres zonas en estudio. La difusividad y conductividad térmica, calor especifico y la densidad de las acerolas frescas fue de $1.4696 \times 10^{-7} \mathrm{~m}^{2} \mathrm{~S}^{-1}, 0.5979 \mathrm{~W} \mathrm{~m}^{-1} \mathrm{C}^{-1}, 3.9685 \mathrm{~kJ} \mathrm{~kg}^{-1}$ ${ }^{\circ} \mathrm{C}^{-1}$ y $1086.6077 \mathrm{~kg} \mathrm{~m}^{-3}$, en lo sensorial los resultados muestran, un comportamiento del sabor semejante para las muestras, excepto en el sabor dulce, en donde la muestra proveniente del Sinú medio logró una diferencia en la intensidad de este atributo.
\end{abstract}

Palabra clave: acerola, Propiedades Termofísicas, análisis sensorial

\section{Composition and Sensory Analysis Thermophysical Colombian Fruits. Part 2: Acerola (Malpighia emarginata L.)}

\begin{abstract}
The objective of this work is the moisture content, carbohydrate and ash in acerola (Malpighia emarginata $\mathrm{L}$ ) in three regions of the department of Córdoba, and analyzation of the thermophysical and sensory properties. The thermophysical properties, such as conductivity, thermal diffusivity, specific heat and density were determined in the fruit according to the chemical composition and comparing acerola DEPROTER software. Ash and moisture values in fruits did not find significant differences in the regions of high and medium Sinu carbohydrates $(\mathrm{p}<0.05)$ in the three study areas. Diffusivity and thermal conductivity, specific heat and density of fresh acerola cherries was $1.4696 \times 10^{-7} \mathrm{~m}^{2} \mathrm{~S}^{-1}, 0.5979 \mathrm{~W} \mathrm{~m}^{-1} \mathrm{C}^{-1}, 3.9685 \mathrm{~kJ} \mathrm{~kg}^{-1}{ }^{\circ} \mathrm{C}^{-1}$ and $1086.6077 \mathrm{~kg} \mathrm{~m}^{-3}$, sensory as the results show similar behavior for sample flavors except sweet taste, which the sample from the Sinu achieved a difference in the intensity of these attributes.
\end{abstract}

Keywords: acerola, thermophysical properties, sensory analysis. 


\section{INTRODUCCIÓN}

La acerola es conocida en el caribe como cereza de Barbados, es originaria de América central e introducida al sur del continente en la década de los años 50 , adaptándose muy bien a las temperaturas de las zonas y a los diferentes tipos de suelos. Su propagación se realiza por semillas presentando una gran variabilidad genética. En estos momentos Brasil es el mayor productor, consumidor y exportador del fruto acerola, produciendo 32990 toneladas, la acerola (Malpighia emarginata L), también conocida como cereza o cherry antillano, está presente en el sur y central Latina, así como en algunas regiones del sur de América del Norte (Johnson, 2003). Es una fruta con forma redonda roja, con un diámetro variando de $1 \mathrm{a} 4 \mathrm{~cm}$, con una piel delgada que se daña fácilmente. Generalmente los frutos de acerola son muy frágiles y sensibles, por lo que necesitan tener un manejo especial y buenas condiciones de almacenamiento. Su demanda se ha incrementado en las últimas décadas, gracias a su alto contenido de ácido ascórbico contenidos, que va 1000 a 4.500 mg/100 g (Leung y Foster, 1996; Mezadri et al., 2006). Freitas et al., (2006) y Mezadri et al., (2008) determinaron azucares totales, humedad y fibra con resultados muy parecidos (10.9) g, 86.5\%, 0.82 g. Las propiedades termofísicas son parámetros importantes en la descripción de la transferencia de calor durante el calentamiento de alimentos sólidos, suscitando grandes ventajas en los costos energéticos, la calidad y la seguridad del alimento (Feyissa 2011; Hassan y Ramaswamy 2011). El calor específico Cp, difusividad térmica a y conductividad térmica $\mathrm{k}$; son las propiedades termofísicas más importantes en el procesamiento de alimentos (Bitra et al., 2010; Feyissa, 2011). Estudios recientes han demostrado que la acerola presenta actividad antioxidante basada en su capacidad de secuestrar radicales libres (Berliner y Heinecke, 1996; siendo así adecuada para la prevención de enfermedades relacionadas con la edad, tales como hipertensión,

El fruto presenta un período de vida corto después de ser recogido (2 a 3 días), a temperatura ambiente, en donde la maduración de la fruta consiste en una serie de complejas bioreacciones químicas como la hidrólisis del almidón, conversión de los cloroplastos en cromoplastos, transformación de la clorofila, la producción de carotenoides, antocianinas y compuestos fenólicos y la formación de compuestos volátiles.

Todas estas reacciones son importantes para el caracterizar las frutas en su estado de madurez aprovechando además para uso industrial su peculiar aroma (Speirs y Brady, 1991).

Las frutas debido a su alto contenido en agua son productos altamente perecederos y posibilitan el desarrollo de una compleja secuencia de reacciones donde participan enzimas, además de los procesos fisiológicos propios de la respiración, transpiración y pérdida de la calidad con el deterioro de vitaminas, de pigmentos y carbohidratos, principalmente (Benedetti et al., 2008). Considerando la actividad de agua de éstos productos y dada la actividad propia de agentes microbianos, se propician el crecimiento de microorganismos, pues se confiere el sustrato adecuado para su evolución. Estos cambios se evidencian durante la etapa poscosecha del producto, principalmente en el almacenamiento (Cocci et al., 2006). El objetivo del presente trabajo fue analizar las propiedades termofísicas y sensoriales del fruto de la acerola (Malphigia emarginata L) y el estudio de contenidos de humedad, carbohidratos y cenizas.

\section{MATERIALES Y MÉTODOS}

\section{Recolección de los frutos}

Los frutos de la acerola fueron recolectados al azar, en árboles existentes en las tres subregiones del Alto, Medio y Bajo Sinú del departamento de Córdoba (Colombia); en la zona del Alto Sinú el área de estudio comprendió el municipio de Valencia, en la zona del Medio Sinú se realizó la recolección en los municipios de Cereté y San Carlos y en la zona del Bajo Sinú se realizaron recolecciones en el municipio de Lorica. En cada región del Alto, Medio y Bajo Sinú se muestrearon 25 árboles aproximadamente.

\section{Obtención de pulpa o jugo}

Los fruto se recolectaron en su punto óptimo de calidad de consumo enteras y sin daños mecánicos se pesaron, seleccionaron, clasificaron, lavaron y se escaldaron a una temperatura de $60^{\circ} \mathrm{C}$ por 15 minutos y se llevaron a la planta piloto donde se le realizó la extracción del jugo mediante una despulpadora tipo Helicoidal Marca Fedeacero Modelo X35T, el jugo se refinó en un tamiz Tyler de $0.2 \mu \mathrm{m}$, este se pasteurizó a $72 \pm 2{ }^{\circ} \mathrm{C}$ por $15 \mathrm{~s}$ en un pasteurizador de placa Alpha Laval ${ }^{\circledR}$, luego se envasó y refrigeró a $4{ }^{\circ} \mathrm{C}$ para su conservación y análisis. Se realizó un análisis sensorial del jugo obtenido, empleando la prueba de medición del grado de satisfacción, en esta prueba afectiva se contó con 25 panelistas no entrenados, cuyas edades estaban comprendidas entre los 16 y 50 años de edad y posibles consumidores. Se evaluó la aceptación o rechazo del producto empleando como instrumento de medición una escala hedónica verbal de 5 puntos. 


\section{Caracterización bromatológica del fruto.}

Se realizó la extracción manual de la pulpa o jugo del envase refrigerado. La caracterización se realizó mediante la utilización de las técnicas del análisis proximal, Humedad: Método 930.15/90 AOAC; Determinación de grasa: Método 920.39/90 AOAC; Determinación Cenizas: Método 942.04/90 AOAC; Determinación Fibra cruda: Método 962.09/90.AOAC; Determinación Proteína Cruda 955.04/90 AOAC y los carbohidratos se determinaron por diferencia.

\section{Análisis Termofísico de la acerola.}

Las propiedades termofísicas, como conductividad térmica, la difusividad térmica, el calor específico y la densidad, se determinaron de acuerdo a la composición bromatológica de la acerola, empleando el modelo matemático más utilizado para conocer estas propiedades que es modelo desarrollado por Choi y Okos (1985) basado en la temperatura y la composición que tiene el alimento, como, humedad, proteína, grasa, fibra, carbohidratos y cenizas y se comparó con el software elaborado por Alvis et al. (2012) para determinar propiedades termofísicas.

\section{Análisis sensorial acerolas frescas}

Los parámetros de selección de las muestras fueron: Acerolas enteras, sanas, frescas y limpias. Color correspondiente a los estados de madurez 5 y/o 6 según NTC 4580:1999.

\section{Determinación de atributos}

Para realizar el perfil sensorial fue necesario identificar y seleccionar los descriptores que caracterizan a esta fruta, esto se realizó a través de los siguientes pasos: La determinación de los descriptores para evaluar acerola, comenzó con la descripción libre de diversas muestras por los jueces entrenados, esto proporcionó un total de 48 descriptores. Se realizó la primera reducción de descriptores con ayuda del líder del panel, intentando eliminar de la lista atributos repetitivos, hedonísticos y cuantitativos. Al finalizar esta reducción se obtuvieron 35 descriptores de sabor y 24 descriptores olor, (flavor) encontrados en las acerolas de diferente origen.

Debido a que el número de descriptores era alto, fue necesario utilizar la metodología planteada por NTC 3932:1996, permitiendo reducir los atributos para obtener un mejor resultado y mayor objetividad al momento de realizar las pruebas descriptivas. Para lograr esto se realizó el cálculo de la media geométrica, a través de la ecuación 1.

$M=(F * I)^{1 / 2}$

Donde $\mathrm{F}$ es igual a la frecuencia y corresponde al número de veces que el descriptor es mencionado sobre el total de veces que puede ser mencionado. I es la intensidad relativa de cada descriptor y corresponde a la suma de las intensidades dadas por el panel sobre la intensidad máxima del descriptor (NTC 3932, 1996). La reducción de los descriptores se realizó eliminando los atributos con un valor menor a 2.5. Las muestras fueron presentadas, enteras con cáscara, a temperatura ambiente (aprox. $30^{\circ} \mathrm{C}$ ), en platos desechables, acompañadas de agua como sustancia de enjuague y el formato de evaluación.

Se empleó la técnica descriptiva conocida como perfil sensorial, esta metodología permite determinar la intensidad de atributos sensoriales, a través de escalas de respuesta estandarizadas (NTC 3929). La valoración de la intensidad de los atributos se realizó a través de una escala de respuesta de 0 a 5 , en donde 0 representa ausencia del atributo y 5 indica una intensidad alta del atributo, las NTC anteriormente enunciadas se utilizan para la evaluación sensorial de frutas frescas NTC 4580 (1999) (Uchuva), para este caso utilizamos las mismas por las características similares de los frutos y no existir una norma validada para la acerola.

\section{Análisis estadístico}

El diseño de la investigación fue un diseño experimental completamente al azar con tres repeticiones por tratamiento, bajo una estructura de tratamiento unifactorial. Las variables independientes fueron las regiones del Alto, Medio y Bajo Sinú y las variables dependientes la constituyeron los porcentajes de humedad, carbohidratos y cenizas. Para cada variable se llevó a cabo un análisis de varianza ANOVA y se utilizó la prueba de Tukey para determinar las diferencias significativas entre las tres regiones estudiadas. 


\section{RESULTADOS Y DISCUSIÓN}

\section{Análisis del contenido de humedad, carbohidratos y cenizas.}

Las variables analizadas del fruto para las tres regiones estudiadas se detallan en la Tabla 1. En la Tabla 2, los resultados son en base seca y las letras diferentes muestran diferencias estadísticamente significativa. El contenido promedio porcentual de constituyentes de la acerola, de las tres regiones alto, medio y bajo Sinú mostraron diferencias significativas $(p<0.05)$, siendo el mayor porcentaje de carbohidratos obtenido en la zona del medio Sinú con un valor del $65.3 \%$ (Tabla 1 ).

Tabla 1. Análisis composicional de acerolas en tres regiones

\begin{tabular}{cccc}
\hline Composicion & Bajo Sinu & Medio Sinu & Alto Sinu \\
\hline Carbohidratos & $56.12 \mathrm{a}$ & $65.31 \mathrm{~b}$ & $54.56 \mathrm{c}$ \\
Humedad & $92.60 \mathrm{a}$ & $93.46 \mathrm{a}$ & $94.98 \mathrm{~b}$ \\
Cenizas & $5.23 \mathrm{a}$ & $4.91 \mathrm{~b}$ & $5.04 \mathrm{~b}$ \\
\hline
\end{tabular}

Los resultados de las variables analizadas como humedad y ceniza no se encontraron diferencias significativas en las frutas provenientes de las zonas del medio y el alto Sinú, sin embargo, se observó diferencias estadísticamente significativas $(p<0.05)$ entre la región alto y medio Sinú con respecto a la región del bajo Sinú. Los porcentajes más altos de humedad fueron los de las frutas provenientes de la región del alto Sinú. En cuanto a la humedad Vendramini y Trugo (2000) obtuvieron resultados muy parecidos a los de esta investigación $92 \%$ y en cenizas por debajo $0.4 \%$.

Las diferencias obtenidas en los contenidos de carbohidratos, ceniza y humedad para las tres zonas en estudio denotan influencia significativa de las condiciones de los suelos, la temperatura, la pluviosidad, la altitud, la textura del suelo, minerales, oligoelementos y otros nutrientes que se encuentra en cada una de las regiones. El contenido de humedad es un factor importante en el tratamiento y manejo postcosecha potencialmente destinadas al procesamiento industrial, debido a que son parámetros importantes en la conservación de la materia prima, (Eltelib et al., 2011)

\section{Análisis termofísico de la acerola fresca}

Las propiedades termofísicas se calcularon a partir de la composición fisicoquímica (Tabla 2) utilizando las ecuaciones de (Choi y Okos, 1985). Los resultados mostrados en la tabla son en base húmeda.

Tabla 2. Composición fisicoquímica del fruto de la acerola.

\begin{tabular}{cc}
\hline Composicion & Acerola $^{*} \%$ \\
\hline Humedad & 92.71 \\
Cenizas & 6.18 \\
Grasa & 0.52 \\
Proteína & 0.17 \\
Fibra & 0.16 \\
Carbohidratos & 0.43 \\
\hline
\end{tabular}

Aunque hoy en día no se encuentran muchos resultados de análisis termofísicos de la acerola, los resultados obtenidos en esta investigación, ayudan a mejorar características fisicoquímicas y organolépticas de productos procesados como mermeladas y compotas, evitando la cristalización de los azúcares mediante la acción de altas temperaturas.

Tabla 3. Propiedades Termofísicas

\begin{tabular}{ccccc}
\hline Propiedad & $k\left(\mathrm{~W} \mathrm{~m}^{-1} \mathrm{C}^{-1}\right)$ & $C_{p}\left(\mathrm{KJ} \mathrm{kg}^{-1}{ }^{\circ} \mathrm{C}^{-1}\right)$ & $\alpha\left(\mathrm{m}^{2} \mathrm{~s}^{-1}\right)$ & $\rho\left(\mathrm{kg} \mathrm{m}^{-3}\right)$ \\
\hline Acerola fresca & 0.5979 & 3.9685 & $1.4696 \times 10^{-7}$ & 1086.6077 \\
\hline
\end{tabular}


Al comparar los valores obtenidos de la ecuación de Choi y Okos (1985) para la difusividad y conductividad térmica, el calor especifico y la densidad de acerolas frescas $1.4697 \times 10^{-7} \mathrm{~m}^{2} \mathrm{~s}^{-1}, 0.5979 \mathrm{~W} \mathrm{~m}^{-1} \mathrm{C}^{-1}, 3.9685 \mathrm{~kJ}$ $\mathrm{kg}^{-1}{ }^{\circ} \mathrm{C}^{-1}$ y $1086.6077 \mathrm{~kg} \mathrm{~m}^{-3}$ respectivamente que se muestra en la tabla 3, con la herramienta DEPROTER elaborado por Alvis et al. (2012) cuyos valores fueron $1.4728 \times 10^{-7} \mathrm{~m}^{2} \mathrm{~s}^{-1}, 0.6063 \mathrm{~W} \mathrm{~m}^{-1} \mathrm{C}^{-1}, 3.9676 \mathrm{~kJ} \mathrm{~kg}^{-1}$ ${ }^{\circ} \mathrm{C}^{-1}$ y $1084.6754 \mathrm{~kg} \mathrm{~m}^{-3}$ respectivamente, no se observaron diferencias significativas. Estos valores son similares con los obtenidos por otros autores en frutos frescos como los reportados por Alvarado (1989) quien obtuvo resultados en el calor específico y conductividad térmica para fresa, melón y tomate muy cercanos a los obtenidos en esta investigación, de $3.82 ; 3,76$ y $3.93 \mathrm{~kJ} \mathrm{~kg}^{-1}{ }^{\circ} \mathrm{C}^{-1}$. Para conductividad y difusividad térmica Alvarado (1994) encontró valores muy cercanos a los reportados en este trabajo para fresa, melón y tomate con valores de $0.52 \mathrm{~W} \mathrm{~m}^{-1}{ }^{\circ} \mathrm{C}^{-1}$ y $1.47 \times 10^{-7} \mathrm{~m}^{2} \mathrm{~s}^{-1} ; 0.57 \mathrm{~W} \mathrm{~m}^{-1}{ }^{\circ} \mathrm{C}^{-1}$ y $1.59 \times 10^{-7} \mathrm{~m}^{2} \mathrm{~s}^{-1}$; $0.55 \mathrm{~W} \mathrm{~m}^{-1}{ }^{\circ} \mathrm{C}^{-1}$ y $1.50 \times 10^{-7} \mathrm{~m}^{2} \mathrm{~s}^{-1}$. Para aguacate y ciruela, Alvarado (1994) encontró valores más bajos a los encontrados en esta investigación con valores en conductividad y difusividad térmica $0.39 \mathrm{~W} \mathrm{~m}^{-1}{ }^{\circ} \mathrm{C}^{-1} \mathrm{y}$ $1.16 \times 10^{-7} \mathrm{~m}^{2} \mathrm{~s}^{-1}$ y para ciruela $0.54 \mathrm{~W} \mathrm{~m}^{-1}{ }^{\circ} \mathrm{C}^{-1}$ y $1.49 \times 10^{-7} \mathrm{~m}^{2} \mathrm{~s}^{-1}$.Biju y Carson (2008) midieron la difusividad en cacahuete (Castanea sativa) a $30^{\circ} \mathrm{C}$ la cual fue de $5.1 \times 10^{-11} \mathrm{~m}^{2} \mathrm{~s}^{-1}$. Asimismo, en grosellas se han reportado difusividad y conductividad térmica de $1.38 \times 10^{-7} \mathrm{~m}^{2} \mathrm{~s}^{-1}$ y $0.22 \mathrm{~W} \mathrm{~m}^{-1} \mathrm{~K}^{-1}$ y $C p$ de $1.64 \mathrm{~kJ} \mathrm{~kg}^{-1}{ }^{\circ} \mathrm{C}^{-1}$ (Gordillo et al., 2009) valores por debajo a esta investigación. La densidad de la acerola fue más alta que la reportada por otros autores; Demir et al. (2003) reportaron una densidad promedio de avellanas de $848 \mathrm{~kg}$ $\mathrm{m}^{-3}$ para almendras tostadas, sin embargo en almendras fresca reporta una densidad de $1150.6 \mathrm{~kg} \mathrm{~m}^{-3}$. Las propiedades termofísicas de frutos tropicales están correlacionados al porcentaje de humedad del producto (Razavi y Taghizadeh, 2007; Bitra et al., 2010). La literatura ha revelado que muy pocas investigaciones se han llevado a cabo en acerolas para determinar el calor específico, conductividad térmica y la difusividad térmica. El conocimiento de las propiedades térmicas en frutos promisorios para la agroindustria, es necesaria para el modelaje, optimización y el diseño de los procesos y equipos de procesamiento en las operaciones basadas en tratamientos térmicos tales como secado, tostado, enfriamiento o congelación (Ariza y Gordillo 2007; Mercali et al., 2013).

\section{Análisis sensorial acerolas frescas}

Como se muestra en la figura 1, el flavor de la acerola, en sus compuestos volátiles, se compone de notas frutales, florales y cítricas Los panelistas relacionaron estos atributos con frutas tropicales presentes en Colombia como el tomate de árbol, lulo y mandarina. Esto se puede explicar por una posible relación en la composición volátil de estas frutas, como el acetato de butilo, limoneno, terpinoleno, octanoato de etilo y acetato de etilo, que proporcionan las notas aromáticas descritas en este trabajo y que se encuentran presentes en estas frutas (Ormaza y Taborda, 2003; Duque y Morales, 2005; Yu et al., 2007; Restrepo et al., 2008). Se evidenció un comportamiento del sabor semejante para las muestras, excepto en el sabor dulce, en donde la muestra proveniente del Sinú medio logró una diferencia en la intensidad de este atributo, esta fue verificada a través de una análisis de varianza, con un valor $p<0.05$.

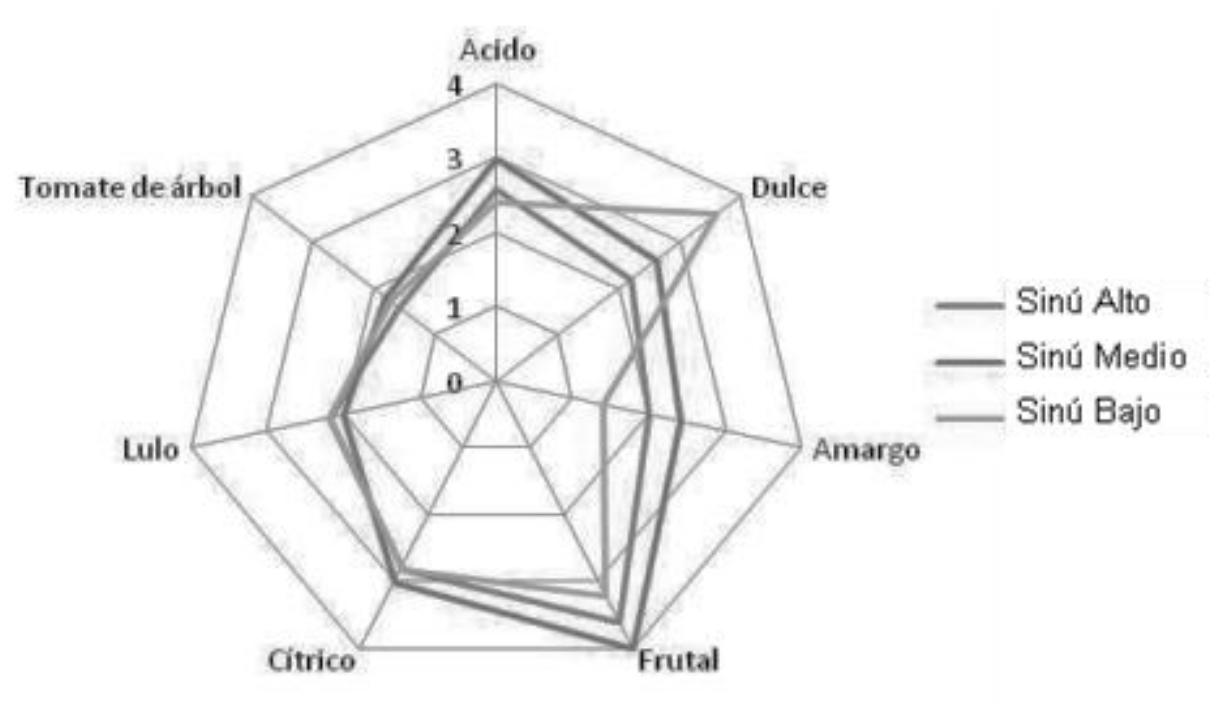

Fig. 1. Perfil sensorial de las tres regiones del Córdoba por el panel de consumidores.

El aroma y sabor de la acerola para las tres regiones presentó similitudes en los descriptores frutal, cítrico y lulo, el atributo mandarina tenía una menor intensidad en la muestra del Sinú medio, y los atributos tomate de árbol y floral se presentaron en mayor intensidad en las muestras de alto y bajo Sinú, respectivamente; sin embargo el análisis estadístico no evidenció diferencias significativas. 


\section{CONCLUSIONES}

El contenido de humedad del fruto de la acerola es dependiente de la región geográfica donde se cultive, sin embargo, solo se encontraron diferencias significativas en dos regiones bajo y medio Sinú contra Alto Sinú en cuanto a carbohidratos se presentaron diferencias significativas en las tres regiones. Con relación a las propiedades termofísicas, las cuales según esta investigación son muy similares a los encontrados por otros autores en frutas frescas del trópico húmedo, en lo sensorial los resultados muestran, un comportamiento del sabor semejante para las muestras, excepto en el sabor dulce, en donde la muestra proveniente del Sinú medio logró una diferencia en la intensidad de este atributo, esta fue verificada a través de una análisis de varianza, con un valor $\mathrm{p}<0.05$.

\section{REFERENCIAS}

Alvarado, J. de D. Determinación de propiedades físicas y térmicas en aceites, jugo, pulpa de hortalizas y frutas cultivadas en Ecuador. Informe final. Proyecto UTA-CONUEP. Ambato, Ecuador (1989).

Alvarado, J. de D. Propiedades físicas de frutas. IV. Difusividad y conductividad térmica efectiva de pulpas. Latin American Applied Research, 24: 41-47 (1994).

Alvis, A., I. Caicedo y P. Peña, Determinación de Propiedades Termofísicas de Alimentos en Función de la Concentración y la Temperatura empleando un Programa Computacional. Inf. tecnol., vol.23, no.1, p.111116. ISSN 0718-0764 (2012).

Ariza, C. H. y D. F. Gordillo, Uso de la Técnica Fotoacústica para la Caracterización de Café Proveniente del Eje Cafetero Colombiano. Rev. Acad. Colomb. Cienc. 31(119): 217-234. (2007).

Biju C. A. Carson, K. Drying curves and apparent diffusivity of New Zealand chestnut variety '1015'. Journal of Food Engineering 85: 381-386. (2008).

Bitra, V., S. Banu, P. Ramakrishna, G. Narender y A. Womac, Moisture dependent thermal properties of peanut pods, kernels, and shells. Biosystems Engineering 106 :506-512. (2010).

Berliner J. y J. W. Heinecke, The role of oxidized lipoproteins in atherogenesis. Free Radic. Biol. Med. 20:707-727. (1996).

Benedetti, S., M. González, E. García y I. Quiroz, An analysis of the physical and germination parameters of the sweet Chestnut (Castanea sativa). Cien. Inv. Agr. 39(1):185-192. (2012)

Cocci, E., P. Rocculi, S. Romani y M. Dalla Rosa, Changes in nutritional properties of minimally processed apples during storage. En: Postharvest Biology and Technology. Vol. 39(3):265-271. (2006).

Choi Y. y M. Okos, Effects of temperature and composition on the thermal properties of foods. Food Engineering and Process Applications 1: 93-101. (1985).

Demir, A. D., P. Baucour, K. Cronina y K. Abodayeh, Analysis of temperature variability during the thermal processing of hazelnuts. Innovative Food Science and Emerging Technologies 4: 69-84. (2003).

Duque C. y A. L. Morales, El aroma frutal de Colombia. Colombia: Universidad Nacional de Colombia. 345 p. 2005.

Eltelib, H., A. Adebanjo, Y. Badejo, Y. Fujikawa y E. Muneharu, Gene expression of monodehydroascorbate reductase and dehydroascorbate reductase during fruit ripening and in response to environmental stresses in acerola (Malpighia glabra). Journal of Plant Physiology 168, 619-627. (2011)

Feyissa, A. H., Modelling of Heat and Mass Transfer in Processing of Solid Foods. Tesis de Ph.D. National Food Institute, Food Production Engineering, Universidad Técnica de Dinamarca. (2011).

Freitas, C., G. Maia, J. M. Correia da Costa, R. Wilane de Figueiredo y P. Machado de Sousa, Acerola: production, composition, nutritional aspects and products. R. Bras. Agrociência, Pelotas, v. 12, n. 4, p. 395400. (2006).

Gordillo D. F., V. A. Ortega y R. F. Zarate, Correlación Entre Parámetros Térmicos de Café de Diferente Origen. Revista Colombiana de Física 41(1). (2009). 
Hassan, H. y H. Ramaswamy, Measurement and targeting of thermophysical properties of carrot and meat based alginate particles for thermal processing applications. Journal of Food Engineering 107: 117-126. (2011).

Johnson, P. D., Plants in human health and nutrition policy. In A. P. Simopoulos, \& C. Gopalan (Eds.) (pp. 67-75). Basel: Karger. (2003).

Leung, A. Y. y S. Foster, Encyclopedia of common natural ingredients used in food, drugs, and cosmetics (2nd ed.). New York: Wiley. 6-7. (1996).

Mezadri, T., M. S. Fernández-Pacho' n, D. Villano, M. C. García-Parrilla, A. M. Troncoso, El fruto de la acerola: composición. características productivas e importancia económica. Archivos Latinoamericanos de Nutricio'n, 56(2). http://www.alanrevista.org/ediciones/2006-2/fruto_acerola.asp. Available at Accessed 12.11.2006. (2006).

Mercali, G. D., D. Jaeschke, I. C. Tessaro y L. Ferreira Marczak, Degradation kinetics of anthocyanins in acerola pulp: Comparison between ohmic and conventional heat treatment. Food Chemistry $136853-857$. (2013).

Ormaza, A. y G. Taborda, Determinación preliminar por cromatografía de gases de los compuestos volátiles presentes en el espacio de cabeza de uchuva (Physalis peruviana L.). Revista de la Universidad de Caldas. 24: 111-122.(2003).

Razavi, S. M. y M. Taghizadeh, The specific heat of pistachio nuts as affected by moisture content, temperature, and variety. Journal of Food Engineering 79: 158-167. (2007).

Restrepo, L., G. Taborda y A. Ormaza, Extracción y caracterización de los componentes volátiles en la pulpa de uchuva (Physalis peruviana L.) por SDE/GC/MS y SPME/GC/MS. 5to Congreso Colombiano de Cromatografía.: Memorias SENSIBER - COCOCRO V. 32. (2008).

Speirs, J. y C. J. Brady, Modication of gene expression in ripening fruit. Australian Journal of Plant Physiology, 18, 519 \pm 532 . (1991).

Vendramini Ana L. y Luiz C. Trugo, Chemical composition of acerola fruit (Malpighia punicifolia L.) at three stages of maturity. Food Chemistry 71 (2000) 195-198. (2000).

Yu, Q., B. Xie y Y. Zhang, Study on aroma components in fruit from three different Satsuma mandarin varieties. Agricultural Sciences in China.6 (12): 1487-1493.(2007). 
